\title{
EQUIVALENCE OF CERTAIN REPRESENTING MEASURES
}

\author{
I. GLICKSBERG ${ }^{1}$
}

\begin{abstract}
If an interior component $\Omega$ of a compact $K \subset \mathrm{C}$ is a part for $R(K)$, then given $z_{1}, z_{2}$ in $\Omega$ and a representing measure $\lambda_{1}$ for $z_{1}$ there is a representing measure for $z_{2}$ equivalent to $\lambda_{1}$.
\end{abstract}

Given two points $\varphi$ and $\psi$ in the same Gleason part of a uniform algebra, and $\lambda$ in $M_{\varphi}$ (the set of representing measures for $\varphi$ ), a well-known result of Bishop [2, p. 143] insures there is a $\mu$ in $M_{\psi}$ which bounds $\lambda$, and indeed there is a pair $\lambda \in M_{\varphi}$, $\mu \in M_{\psi}$ which mutually bound one another. However it is not in general the case that each $\lambda$ in $M_{\varphi}$ is equivalent to some element of $M_{\psi}$, and our purpose is to point out one instance where that occurs.

Recall that for $K \subset \mathrm{C}$ compact, $R(K)$ is the uniform closure in $C(K)$ of the rational functions.

TheOREM. Suppose $K \subset \mathrm{C}$ is compact and $\Omega$ is a component of the interior $K^{\circ}$ which is also a Gleason part for $R(K)$. Then for $z_{1}, z_{2} \in \Omega$ and $\lambda_{1} \in M_{z_{1}}$ there is $a$ $\lambda_{2} \in M_{z_{2}}$ equivalent to $\lambda_{1}$; moreover for any nonzero measure $\mu$ on $\partial K$ orthogonal to $R(K)$ with $\mu \ll \lambda_{1}$ there is a $\lambda \in M_{z_{2}}$ equivalent to $|\mu|$.

Here $M_{z}$ is the set of representing measures on $\partial K$, as usual. Of course the first conclusion fails if the part containing the component $\Omega$ contains a boundary point since no measure representing $z \in \Omega$ is equivalent to a point mass; and it could only extend to a part consisting of several interior components if these all shared the same boundary (since $\partial \Omega$ is always the topological support of harmonic measure for $z \in \Omega$ ). Consequently it seems unlikely that it holds for any noncomponent parts.

It is worth noting that the result is not due to all elements of $M_{z}$ being mutually equivalent; an example is provided by the champagne bubble set $K$ (given in [5, 27.6]) built from Beurling's function by McKissick in constructing his regular uniform algebra: there $K$ consists of the unit disc $D$ less disjoint subdiscs of finite total circumference converging to $\partial D, K^{\circ}$ is dense and connected, and there is an $f \in R(K)$ with $f^{-1}(0)=\partial D$. Consequently for $z \in K^{\circ}, \partial D$ is necessarily a Jensen null set (so of harmonic measure $\lambda_{z}$ zero); but via Cauchy $d z$ provides an orthogonal measure $\mu$ on $\partial K$, which is absolutely continuous with respect to some $\lambda \in M_{z}$ by the known decomposition of orthogonal measures [5, 23.6] and Wilken's theorem [2, p. 47], and $\lambda$ and $\lambda_{z}$ are inequivalent.

Received by the editors September 14, 1979 and, in revised form, July 9, 1980.

1980 Mathematics Subject Classification. Primary 46J10, 30 A98.

${ }^{1}$ Work supported in part by the NSF.

() 1981 American Mathematical Society 0002-9939/81/0000-0312/\$01.75 
Our result is a simple consequence of an abstract F. and M. Riesz theorem for bands due to Brian J. Cole (unpublished) and König and Seever [4] which we state in a form convenient for our use (cf. [1, 3.1, p. 31]) for a uniform algebra $A$ on $X$ (= $\partial K$ in our application).

TheOREM (COLE-KÖNIG-SEEVER). Let $m$ be a probability measure on $X$ and suppose $M_{\varphi}(m)=M_{\varphi} \cap L^{1}(m) \neq \varnothing$. If $\mu \ll m$ is a measure orthogonal to $A$ and we choose ${ }^{2} \lambda$ in $M_{\varphi}(m)$ so that $\left\|\mu_{\lambda}\right\|$ is a maximum, where $\mu_{\lambda}$ is the component of $\mu$ absolutely continuous with respect to $\lambda$, then $\mu_{\lambda} \perp A$.

(Here $L^{1}(m)$ is our band [1], and $\mu=\mu_{\lambda}+\mu_{\lambda}^{\prime}$, where $\mu_{\lambda}^{\prime}$ is the component of $\mu$ singular with respect to $\lambda$, is our band decomposition of $\mu$.)

We first apply this F. and M. Riesz theorem to the uniform algebra setting. (I am indebted to the referee for considerable simplification in what follows.)

Proposition. Let $B$ be a uniform algebra on $X$, and let $g \in B$ be nonvanishing on $X$, with $1 / g \in B$ while $C(X)=[B, 1 / g]$, the closed algebra generated by $B$ and $1 / g$. Trivially then $A=C+g B$ is a closed subalgebra of $C(X)$ with $g B$ a maximal ideal, the kernel of a multiplicative linear functional $\varphi$ on $A$.

Suppose $\mu \perp B, \mu \neq 0$. Then there is a representing measure $\lambda$ for $\varphi$ which is equivalent to $|\mu|$.

To begin, since $\mu \neq 0$ and $C(X)=[B, 1 / g]$, there is a least integer $n>1$ with $\mu \perp g^{-n} B$, so $g^{-n} \mu \perp g B$ while $g^{-n} \mu(f)=1$ for some $f \in B$. Thus $f g^{-n} \mu$ is a complex measure representing $\varphi$ on $A=\mathbf{C}+g B$, and so dominates a true (i.e., $\geqslant 0$ ) representing measure $\lambda$ for $\varphi\left[2\right.$, p. 33], and $\lambda \in M_{\varphi}(|\mu|)=M_{\varphi} \cap L^{1}(|\mu|)$.

Now in $M_{\varphi}(|\mu|)$ we can find an element $\lambda$ which is maximal in the sense that for any other element $\lambda^{\prime}$ we have $\lambda^{\prime} \ll \lambda$, and this dominating $\lambda$ clearly has the property that it maximizes $\left\|(b \mu)_{\lambda}\right\|$ for any fixed bounded function $b$, where $(b \mu)_{\lambda}$ is the component of $b \mu \ll \lambda$. So for $b \in B$ if we apply the Cole-König-Seever theorem to the measure $b \mu \perp A$ we have $(b \mu)_{\lambda}^{\prime}=b \mu_{\lambda}^{\prime} \perp A$, where the prime indicates the $\lambda$-singular component. In particular $\mu_{\lambda}^{\prime}(b)=0$ for all $b \in B$, so $\mu_{\lambda}^{\prime} \perp B$.

But if $\mu_{\lambda}^{\prime} \neq 0$, as in the first paragraph we obtain a representing measure $\lambda^{\prime}$ for $\varphi$ on $A$ with $\lambda^{\prime} \ll\left|\mu_{\lambda}^{\prime}\right|$, hence singular with respect to $\lambda$, and clearly $\left(\mu_{\lambda}^{\prime}\right)_{\lambda^{\prime}}$, the component of $\mu_{\lambda}^{\prime} \ll \lambda^{\prime}$, is nonzero; since

$$
\left\|\mu_{\left(\lambda+\lambda^{\prime}\right) / 2}\right\| \geqslant\left\|\mu_{\lambda}\right\|+\left\|\left(\mu_{\lambda}^{\prime}\right)_{\lambda^{\prime}}\right\|>\left\|\mu_{\lambda}\right\|
$$

contradicts the maximality of $\left\|\mu_{\lambda}\right\|$ we conclude $\mu_{\lambda}^{\prime}=0$. Thus $\mu=\mu_{\lambda} \ll \lambda$, and $\lambda$ and $|\mu|$ are equivalent as asserted.

COROllary. Let $K \subset \mathrm{C}$ be compact and suppose the interior $K^{\circ}$ is connected and dense in $K$. Let $\mu$ be a measure on $\partial K$ with $\mu \perp R(K)$, and let $z \in K^{\circ}$. Then there is a representing measure $\lambda$ for $z$ on $R(K)$ which is equivalent to $|\mu|$.

Here we apply the proposition to $X=\partial K$ and the uniform algebra $B=R(K)$ on $X$, with $g(\zeta)=\zeta-z$. We have $A=R(K)=B$ and $\varphi$ evaluation at $z$, and by

\footnotetext{
${ }^{2}$ If $\left\|\mu_{\lambda_{n}}\right\| \rightarrow$ maximum then $\lambda=\Sigma_{1}^{\infty} 2^{-n} \lambda_{n}$ provides such a maximizing $\lambda$.
} 
Runge's theorem $[R(K), 1 / g]=R(\partial K)$. Since every point of $\partial K$ lies in the closure of the connected interior $K^{\circ}$ we know it is a peak point for $R(\partial K)$, and so $R(\partial K)=C(\partial K)$ by Bishop's theorem [2, p. 54]; thus the proposition applies to yield its corollary.

We can now obtain our theorem from the corollary. Note that its first assertion follows from the second by setting $\mu=\left(g-z_{1}\right) \lambda_{z_{1}}$; so we only need to see any $\mu$ on $\partial K$ orthogonal to $R(K)$ with $\mu$ absolutely continuous with respect to a representing measure $\lambda_{0}$ for some point in $\Omega$ is equivalent, for any $z$ in $\Omega$, to some $\lambda \in M_{z}$. But our $\mu$ necessarily has, for $z \notin \Omega^{-}$, a Cauchy transform [2, p. 46] $\hat{\mu}(z)$ which must vanish: if $\hat{\mu}(z) \neq 0$ for $z \notin \Omega^{-}$then $|\mu|$ dominates some representing measure $\lambda$ for $z$, by an old result of Bishop, so since $\lambda_{0}$ and $\lambda$ are not mutually singular, $z$ would lie in the part $\Omega$. Since $\hat{\mu}(z)=0$ for all $z \notin \Omega^{-}$implies $\mu \perp R\left(\Omega^{-}\right)$, while $\Omega^{-}$ necessarily has its interior a union of components of $K^{\circ}$, hence just $\Omega$, for $z \in \Omega$ we can apply the corollary to $\Omega^{-}$to obtain a representing measure $\lambda$ for $z$ on $R\left(\Omega^{-}\right)$ equivalent to $|\mu|$. Trivially $\lambda$ represents $z$ on $R(K)$, so we are done.

In fact the theorem holds with $R(K)$ replaced by $A(K)$, or any $T$-invariant closed subalgebra [3] $A_{0}$ of $C(K)$ lying between $R(K)$ and $A(K)$; the proof is essentially the same since, for such an algebra, $A_{0}=\mathrm{C}+\left(z-z_{0}\right) A_{0}$ (for $\left.z_{0} \in \Omega\right)$, while $K$ is the spectrum and $\partial K$ the Šilov boundary. (In place of applying the corollary one can appeal to the proposition applied to $B=\left(A_{1} \mid \Omega^{-}\right)^{-}=\mathbf{C}+g B, g(\zeta)=\zeta-z$, noting that $[B, 1 / g] \supset R(\partial \Omega)=C(\partial \Omega)$.)

\section{REFERENCES}

1. K. Barbey and H. König, Abstract analytic function theory and Hardy algebras, Lecture Notes in Math., vol. 593, Springer-Verlag, Berlin and New York, 1977.

2. T. W. Gamelin, Uniform algebras, Prentice-Hall, Englewood Cliffs, N.J., 1969.

3. __ Uniform algebras on plane sets, approximation theory, Proc. Internat. Sympos. (Univ. Texas, 1973), Academic Press, New York, 1973, pp. 101-149.

4. H. König and G. L. Seever, The abstract F. and M. Riesz theorem, Duke Math. J. 36 (1969), 791-797.

5. E. L. Stout, The theory of uniform algebras, Bogden and Quigley, Tarrytown on Hudson, N. Y., 1971.

Department of Mathematics, University of Washington, Seattle, Washington 98195 